\title{
Simultaneous Space-Time Discretization for Controlling the Motion of Rigid Bodies Actuated Through Elastic Ropes
}

\section{Timo Ströhle and Peter Betsch}

\author{
Institute of Mechanics \\ Karlsruhe Institute of Technology (KIT) \\ Otto-Ammann-Platz 9, 76131 Karlsruhe, Germany \\ timo.stroehle@kit.edu, peter.betsch@kit.edu
}

\begin{abstract}
This contribution deals with the feedforward control of rigid bodies actuated through elastic ropes. After introducing a method which enables a stable inversion process of the dynamics of a geometrically exact rope, a strategy to solve the cooperative control of a rigid body through multiple ropes is presented.
\end{abstract}

Keywords: Flexible multibody system, Cooperative control, Inverse dynamics

\section{INTRODUCTION}

The inverse dynamics of flexible mechanical systems is concerned with searching forces acting on this system such that a finite number of selected points of this system follow a prescribed motion. One subclass of such systems are ropes for large elastic deformations which can be seen as a onedimensional continuum. Here the aim is to find a force which acts at one end of the rope, such that the other end follows a prescribed trajectory (see Fig. 2). In this connection, the end of the rope might be attached to a mass point or connected to a rigid body.

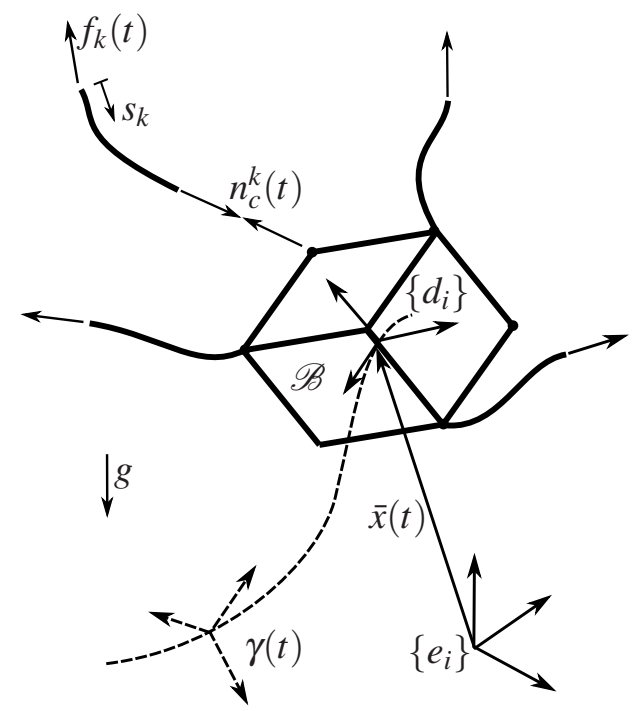

Figure 1. Cooperative transport of a rigid body through $k \in \mathbb{N}$ elastic ropes only actuated through the forces $f_{k}(t)$ at $s_{k}=0$ such that the prescribed motion of the rigid body is realized

After introducing a geometrically exact model for ropes in Section 2, a space-time finite element method based on a simultaneous space-time discretization of the problem at hand is established which solves the inverse dynamics problem (see [1,2]). In Section 3, first the rigid body is introduced as a Cosserat point subjected to geometric constraints. Subsequently, after the inverse dynamics of the rigid body has been discussed, a strategy to solve the cooperative control problem consisting of the rigid body controlled through several elastic ropes undergoing large deformations 
is presented (see Fig. 1). In particular, the cascade-like solution strategy of the inverse multibody system is addressed. The structure of the equations of motion of a multibody system comprised of geometrically exact ropes and rigid bodies is outlined in Section 4. A representative numerical example of the inverse dynamics problem under consideration is presented in Section 5. Eventually, conclusions are drawn in Section 6.

\section{GEOMETRICALLY EXACT ROPES}

The motion of elastic ropes with mass-density $\rho$, length $L$, cross sectional area $A$ and Young's modulus $E$ (see Fig. 2) undergoing large deformations can be described in terms of the arc-length $s \in S=[0, L] \subset \mathbb{R}$ by quasi-linear hyperbolic partial differential equations. For simplicity and without loss of generality, in a stress-free reference configuration, the length of the rope and the cross sectional area is set to $L_{0}=1$ and $A_{0}=1$ respectively. For this, an arbitrary configuration of the rope can be described by the function $r(s, t): S \times T=\Omega \subset \mathbb{R}^{2} \mapsto \mathbb{R}^{\alpha}$ for all $\alpha \in\{1,2,3\}$ and $t \in T=[0, \infty)$. Following [3] the governing equations of motion can be established as follows. With the normal force in the rope $n(s, t): \Omega \mapsto \mathbb{R}^{\alpha}$ and the body-forces $b(s, t): \Omega \mapsto \mathbb{R}^{\alpha}$, balance of linear momentum of an infinitely small piece of rope yields:

$$
\partial_{s} n(s, t)+b(s, t)=\rho \partial_{t}^{2} r
$$

Strings are per definition perfectly flexible e.g. normal forces are strictly tangential to the line of centroids

$$
\partial_{s} r(s, t) \times n(s, t)=0
$$

Introducing the stretch

$$
v(s, t)=\left\|\partial_{s} r(s, t)\right\|
$$

then there exists a $N(s, t): \Omega \mapsto \mathbb{R}$ such that

$$
n(s, t)=N(s, t) v^{-1} \partial_{s} r
$$

After assuming the following constitutive relation

$$
N(s, t)=\frac{E}{2}\left(v-v^{-1}\right)
$$

and introducing the coefficients

$$
A=\rho, \quad B=N(s, t) v^{-1}, \quad C=b(s, t)
$$

the motion of elastic ropes can be described by the following partial differential equation:

$$
A(r, s, t) \partial_{t}^{2} r(s, t)-\partial_{s}\left(B(r, s, t) \partial_{s} r(s, t)\right)=C(r, s, t) \quad \forall \quad(s, t) \in \Omega
$$

Note, that due to the quasi-linearity of the problem at hand, the coefficients $A \in \mathbb{R}, B \in \mathbb{R}$ and $C \in \mathbb{R}^{\alpha}$ may depend on the space and time variables as well as on the solution $r(s, t): S \times T=$ $\Omega \subset \mathbb{R}^{2} \mapsto \mathbb{R}^{\alpha}$ for time $t \in T=[0, \infty)$. To solve the PDE at hand uniquely the following initial

$$
r(s, 0)=r_{0}(s), \partial_{t} r(s, 0)=v_{0}(s) \quad \forall \quad s \in S
$$

and boundary conditions

$$
B \partial_{s} r(0, t)=f(t), B \partial_{s} r(1, t)=n_{c}(t), r(1, t)=\gamma(t) \quad \forall \quad t \in T
$$

need to be defined. Herein $f(t): T \mapsto \mathbb{R}^{\alpha}$ is the searched actuating force at $s=0$ such that the rope at $s=1$ follows the prescribed trajectory $\left(r(1, t)=\gamma(t)\right.$. And $n_{c}(t): T \mapsto \mathbb{R}^{\alpha}$ is the contact 
force at $s=1$. If a mass point $m_{T}$ is attached to the rope at $s=1$, the contact force can be directly computed from the prescribed trajectory of the rope at $s=1$, which coincides with the trajectory of the mass point, via

$$
n_{c}(t)=m_{T}\left(\partial_{t}^{2} \gamma(t)+g e_{z}\right)
$$

Now, the initial boundary value problem constituting the control problem at hand can be solved by applying appropriate numerical procedures. After addressing issues arising from the common semi-discrete approach, where the initial boundary problem at hand is solved by applying the finite element method and subsequently integrating the resulting ordinary differential equation subjected to the given servo-constraint in time by appropriate time-stepping schemes, a space-time finite element method based on a simultaneous space-time discretization is proposed.

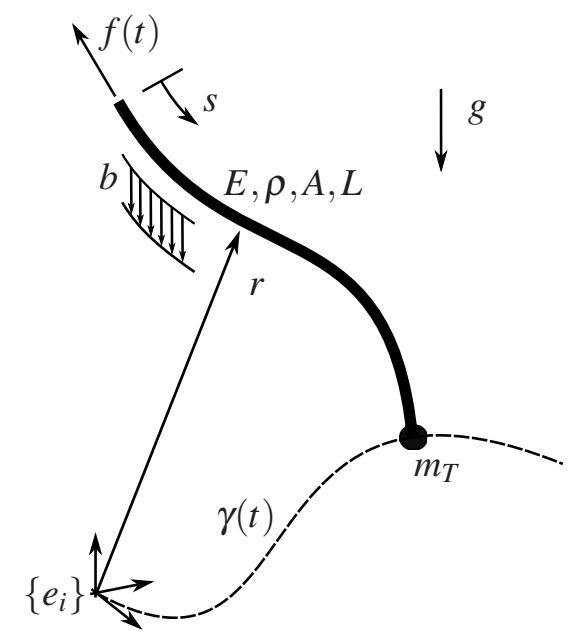

Figure 2. Illustration of the elastic rope for large deformations only actuated by $f(t)$ at $s=0$ such that the rope at $s=1$ follows the prescribed trajectory $\gamma(t)$

\subsection{Semi-discrete equations of motion}

As mentioned above, one common way of solving mechanical problems which are leading to hyperbolic problems is to transform the partial differential equation at hand into a system of ordinary differential equations. Therefore, equation (6) is multiplied with a sufficiently smooth test function and integrated over the spatial domain $s$ :

$$
\int_{S} w \cdot \partial_{s}\left(B \partial_{s} r\right) d s+\int_{S} w \cdot C d s=\int_{S} w \cdot A \partial_{t}^{2} r d s
$$

Integrating the first integral on the left side by parts

$$
\int_{S} w \cdot \partial_{s}\left(B \partial_{s} r\right) d s=\left[w \cdot\left(B \partial_{s} r\right)\right]_{S}-\int_{S} \partial_{s} w B \partial_{s} r d s
$$

leads together with the boundary conditions

$$
B \partial_{s} r(s=0)=f(t), \quad B \partial_{s} r(s=1)=0
$$

to a weak formulation of the problem at hand:

$$
\int_{S} w \cdot A \partial_{t}^{2} r d s+\int_{S} \partial_{s} w B \partial_{s} r d s=\int_{S} w \cdot C d s+\left[w \cdot\left(B \partial_{s} r\right)\right]_{S}
$$


Approximation of the test and trial functions with piecewise continuous Lagrangian polynomials $L_{i}$ :

$$
r^{h}(s)=\sum_{j=1}^{p+1} L_{j}(s) r_{j} ; \quad w^{h}(s)=\sum_{i=1}^{p+1} L_{i}(s) w_{i}
$$

yields with the unknown variables

$$
\hat{q}=\left[\begin{array}{c}
r_{1} \\
\vdots \\
r_{p+1}
\end{array}\right]
$$

the following semi-discrete equations of motion:

$$
\hat{M} \hat{q}+\hat{F}^{i n t}=\hat{N}^{1}-\hat{N}^{0}+\hat{G}
$$

with the components of the quantities given in (11)

$$
\begin{aligned}
& \hat{M}_{i j}=\int_{S} L_{i}(s) A^{h} L_{j}(s) d s \\
& \hat{F}_{i}^{i n t}=\int_{S} \partial_{s} L_{i}(s) B^{h} \partial_{s} L_{j}(s) d s \partial_{s} r_{j} \\
& \hat{G}_{i}=\int_{S} L_{i}(s) C^{h} d s \\
& \hat{N}_{i}^{1}=\delta_{i, p+1} n_{c}(t) \\
& \hat{N}_{i}^{0}=\delta_{i, 1} f(t)
\end{aligned}
$$

By introducing the control condition $r(s=1, t)-\gamma(t)=0$, the control problem can be formulated in terms of the resulting differential algebraic system of equations (DAE). Unfortunately, the differentiation index of the DAE at hand as well as the demands on the differentiability of the given trajectory depend on the spatial discretisation (cf. [4] and [5]). This significantly restricts the applicability of the semi-discrete approach. Therefore a method based on the characteristics of the PDE and a space-time finite element method has been established. Both methods are searching for the solution of the control problem at hand in space and time simultaneously (see [1] and [2] for more details). In this contribution, the space time finite element method is used for computing the inverse dynamics of the problem at hand.

\subsection{Space-time finite element method}

Due to the highly restrictive applicability of solving the control problem at hand sequentially in time, the problem will now be solved simultaneously in space and time. Therefore a spacetime finite element method will be presented in the following (cf.[6],[7],[8]). By introducing the velocity $v(s, t)=\partial_{t} r(s, t)$ the underlying partial differential equation at hand (6) can be transformed into the following system of equations:

$$
\begin{gathered}
\partial_{t} r-v=0 \\
A \partial_{t} v-\partial_{s}\left(B \partial_{s} r\right)=C
\end{gathered}
$$

Multiplying each equation in (17) with sufficiently smooth test functions $w_{1}(s, t)$ and $w_{2}(s, t)$ and integrating over the space-time domain $\Omega=S \times T$ yields the following weak formulation:

$$
\begin{aligned}
\int_{\Omega} w_{1} \cdot\left(\partial_{t} r-v\right) d \Omega & =0 \\
\int_{\Omega} w_{2} \cdot\left(A \partial_{t} v-\partial_{s}\left(B \partial_{s} r\right)\right) d \Omega & =\int_{\Omega} w_{2} \cdot C d \Omega
\end{aligned}
$$


Integrating the second term in (19) by parts

$$
\int_{\Omega} w_{2} \cdot \partial_{s}\left(B \partial_{s} r\right) d \Omega=\int_{T}\left[w_{2} \cdot B \partial_{s} r\right]_{s=0}^{1} d t-\int_{\Omega} \partial_{s} w_{2} \cdot B \partial_{s} r d \Omega
$$

the following equation for (19) can be established:

$$
\int_{\Omega} w_{2} \cdot A \partial_{t} v d \Omega-\int_{T}\left[w_{2} \cdot B \partial_{s} r\right]_{s=0}^{1} \mathrm{~d} t+\int_{\Omega} \partial_{s} w_{2} \cdot B \partial_{s} r d \Omega=\int_{\Omega} w_{2} \cdot C d \Omega
$$

Additionally the servo-constraint $r(s=1, t)=\gamma(t)$ can be demanded weakly on the boundary $\partial \Omega_{\gamma}=\{1\} \times T$

$$
\int_{\partial \Omega_{\gamma}} w_{3}(t) \cdot(r(1, t)-\gamma(t)) d t=0
$$

The task is now to find the unknown functions

$$
\begin{gathered}
r(s, t) \in V_{1}=\left\{r: \Omega \mapsto \mathbb{R}^{\alpha} \mid r\left(\partial \Omega_{0}\right)=r_{0}\right\} \\
v(s, t) \in V_{2}=\left\{v: \Omega \mapsto \mathbb{R}^{\alpha} \mid v\left(\partial \Omega_{0}\right)=v_{0}\right\} \\
f(t) \in V_{3}=\left\{f: \partial \Omega_{f} \mapsto \mathbb{R}^{\alpha} \mid f\left(\partial \Omega_{\gamma} \cap \partial \Omega_{0}\right)=f_{0}\right\}
\end{gathered}
$$

such that for arbitrary but sufficiently smooth test functions

$$
\begin{gathered}
w_{1}(s, t), w_{2}(s, t) \in W_{1}=\left\{w_{1}, w_{2}: \Omega \mapsto \mathbb{R}^{\alpha} \mid w_{1}\left(\partial \Omega_{0}\right)=0, w_{2}\left(\partial \Omega_{0}\right)=0\right\} \\
w_{3}(t) \in W_{2}=\left\{w_{3}: \partial \Omega_{\gamma} \mapsto \mathbb{R}^{\alpha} \mid w_{3}\left(\partial \Omega_{\gamma} \cap \partial \Omega_{0}\right)=0\right\}
\end{gathered}
$$

the equations (18), (21) and (22) are satisfied together with the Neumann boundary conditions

$$
B \partial_{s} r\left(\partial \Omega_{f}\right)=f(t) \quad \text { and } \quad B \partial_{s} r\left(\partial \Omega_{\gamma}\right)=n_{c}(t) \quad t \in T
$$

and the Dirichlet boundary conditions

$$
r\left(\partial \Omega_{0}\right)=r_{0}(s) \quad \text { and } \quad \partial_{t} r\left(\partial \Omega_{0}\right)=v_{0}(s) \quad s \in S
$$

The weak formulation consisting of (18), (21) and (22) subjected to the given Neumann and Dirichlet boundary conditions can then be solved numerically using the finite element method based on a piecewise continuous approximation.

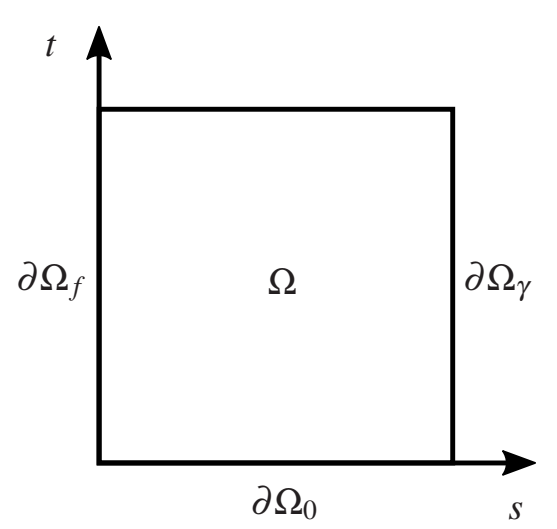

Figure 3. Space-Time domain $\Omega=S \times T$ with the boundaries $\partial \Omega_{0}=S \times\{0\}, \partial \Omega_{f}=\{0\} \times T$ and $\partial \Omega_{\gamma}=\{1\} \times T$ 


\section{RIGID BODY}

When considering general rigid bodies, in principle the same strategy as for the attached mass point can be applied. The actuating forces needed to achieve the desired motion of the rigid body can be calculated directly from the governing equations of motion. These forces can then be inserted into the Neumann boundary condition of the control problem (8). In this contribution a Cosserat point subjected to geometric constraints is used to describe the motion of a rigid body with density $\rho_{0}: \mathscr{B}_{0} \mapsto \mathbb{R}$ and total mass $\bar{M}=\int_{\mathscr{B}_{0}} \rho_{0} \mathrm{dV}$ (see [9] for more details). Accordingly, the equations of motion can be written as

$$
\begin{aligned}
\bar{M} \partial_{t}^{2} \bar{x}-f_{e x t}-G & =0 \\
E_{i j} \partial_{t}^{2} d_{j}-f_{e x t}^{i}+\Lambda_{i j} d_{j} & =0 \\
g_{c}\left(d_{i}\right)=d_{i} \cdot d_{j}-\delta_{i j} & =0
\end{aligned}
$$

Herein $G=\bar{M} g e_{z}$ is the gravitational force and $E_{i j}$ are the components of the referential Euler tensor which is closely related to the classical inertia tensor of rigid body dynamics. Furthermore, $f_{\text {ext }}$ is the resultant external force and

$$
f_{\text {ext }}^{i}=X_{i}^{k} n_{c}^{k}
$$

are the external director forces (Fig. 4). This holds for a discrete actuation of the rigid body. Note that by introducing the matrix

$$
H_{j k}=\left[\begin{array}{c}
I \\
X_{1}^{k} I \\
X_{2}^{k} I \\
X_{3}^{k} I
\end{array}\right]
$$

containing the information of the geometric position of the contact points the following linear relation between the external (director) forces $\bar{f}(t)=\left[\begin{array}{ll}f_{\text {ext }} & f_{\text {ext }}^{i}\end{array}\right]^{T}$ and the contact forces $n_{c}^{k}$

$$
\bar{f}(t)=H n_{c}^{k}(t)
$$

holds. The geometric constraints (23) $)_{3}$ are enforced by the Lagrange multipliers $\Lambda_{i j}$. To force the rigid body at hand to follow a prescribed motion $\gamma(t)=\left[\begin{array}{ll}\gamma_{\bar{x}} & \gamma_{d_{i}}\end{array}\right]^{T}$ additionally to the holonomic constraints $(23)_{3}$, the following control constraints

$$
g_{s}=\bar{q}-\gamma(t)=0
$$

are introduced. In (27) the motion of the rigid body, which is fully described by the directors $d_{i}: T \mapsto \mathbb{R}^{3}$ and the position of the centre of gravity $\bar{x}: T \mapsto \mathbb{R}^{3}$ is contained in $\bar{q}=\left[\begin{array}{ll}\bar{x} & d_{i}\end{array}\right]^{T}$. The servo-constraints (27) of course must not violate the holonomic constraints (23) 3 . The differential part of the DAE at hand, consisting of $(23)_{1}$ and $(23)_{2}$, together with the control constraint (27) yield an algebraic equation for the external (director) force $\bar{f}(t)$ conjugate to $q$ given by

$$
\bar{f}(t)=D \partial_{t}^{2} \gamma(t)+F \gamma(t)-\bar{G}
$$

with

$$
D=\left[\begin{array}{cc}
M & 0 \\
0 & E_{i j}
\end{array}\right] \quad, \quad F=\left[\begin{array}{cc}
0 & 0 \\
0 & \Lambda_{i j}
\end{array}\right] \quad, \quad \bar{G}=\left[\begin{array}{c}
G \\
0
\end{array}\right]
$$

where $G$ is the gravitational force. After $\bar{f}(t)$ has been computed, the $k \in \mathbb{N}$ contact forces $n_{c}^{k}(t)$ for the $k$ ropes at $s_{k}=1$ can be easily computed by knowing the position of the contact point of the rope at the rigid body through the following linear relation:

$$
n_{c}^{k}(t)=Q_{k l} \bar{f}_{l}(t)
$$


Herein $Q_{k l}$ are the components of the inverse of the positive definite matrix $H \in \mathbb{R}^{4 \alpha, 4 \alpha}$ introduced in (25). Once the forces $n_{k}(t)$ have been calculated, each rope can be solved separately by inserting the forces into the corresponding boundary condition of the quasi-linear hyperbolic partial differential equation established in Section 2. Before the cascade-like approach outlined above is investigated numerically in Section 5, the multibody system at hand consisting of a rigid body attached to a geometrically exact rope is developed in the next Section. The numerical results of the inverse calculation can then be verified using this model.

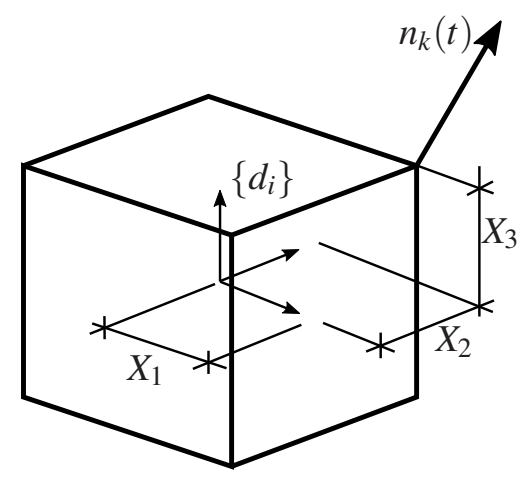

Figure 4. Force $n_{k}$ acting on a rigid body at point $P=\bar{x}+X_{i} d_{i}$

Remark 3.1 (Lagrange multipliers). The actuating forces depend on the Lagrange multipliers $\Lambda_{i j}$ and hence a unique solution for the actuation of the rigid body requires the six independent components $\bar{\lambda}_{k}$ for $k \in\{1, \cdots, 6\} \subset \mathbb{N}$ of $\Lambda_{i j}$ to be partly specified.

$$
\bar{\lambda}_{k}=\gamma_{\lambda_{k}}
$$

In essence, this amounts to partly specifying the stresses within the rigid body (cf.[10]).

\section{MULTIBODY SYSTEM}

When considering flexible multibody systems consisting of a rigid body connected to a rope, the motion can be described by the semi-discrete equation of motion of the rope (11) together with the differential algebraic equation (23) governing the motion of the rigid body depending on the unknown spatial discrete variables $q=\left[\begin{array}{ll}\hat{q}^{T} & \bar{q}^{T}\end{array}\right]^{T}$.

$$
R\left(\partial_{t}^{2} q, q, \bar{\lambda}, t\right)=\left[\begin{array}{c}
\hat{M} \partial_{t}^{2} \hat{q}+f_{i}^{i n t}-\hat{G} \\
D \partial_{t}^{2} \bar{q}+F \bar{q}-\bar{G}
\end{array}\right]
$$

subjected to the following geometric contact constraint

$$
g_{k}=r_{p+1}-\left(\bar{x}+X_{i} d_{i}\right)=0
$$

by the following semi-explicit differential algebraic equation:

$$
\begin{aligned}
R\left(\partial_{t}^{2} q, q, t\right)-\left(\partial_{q} g_{k}(q)\right)^{T} \lambda & =0 \\
g_{c} & =0 \\
g_{k} & =0
\end{aligned}
$$

Note that the Lagrange multipliers $\lambda: T \mapsto \mathbb{R}^{\alpha}$ can be identified with the contact force acting 
between the rope and the rigid body. Compare therefore (11) and (23) with

$$
\lambda \partial_{q} g(q)=\left[\begin{array}{c}
0 \\
\vdots \\
\lambda \\
-\lambda \\
-\lambda X_{1} \\
-\lambda X_{2} \\
-\lambda X_{3}
\end{array}\right]
$$

To verify the numerical implementation of the procedure outlined in this section, an example for the forward dynamics of a rigid body suspended by a flexible rope is considered. In particular, snapshots of a free oscillation of a rigid cube with edge length $a$ and total mass $\bar{M}$, which is attached to a flexible rope, are shown in Figure 5. For the time $t=0.0 \mathrm{~s}$ the initial translational velocity $v_{0}=\left[\begin{array}{lll}2 & -5 & 0\end{array}\right]^{T}$ is presupposed.

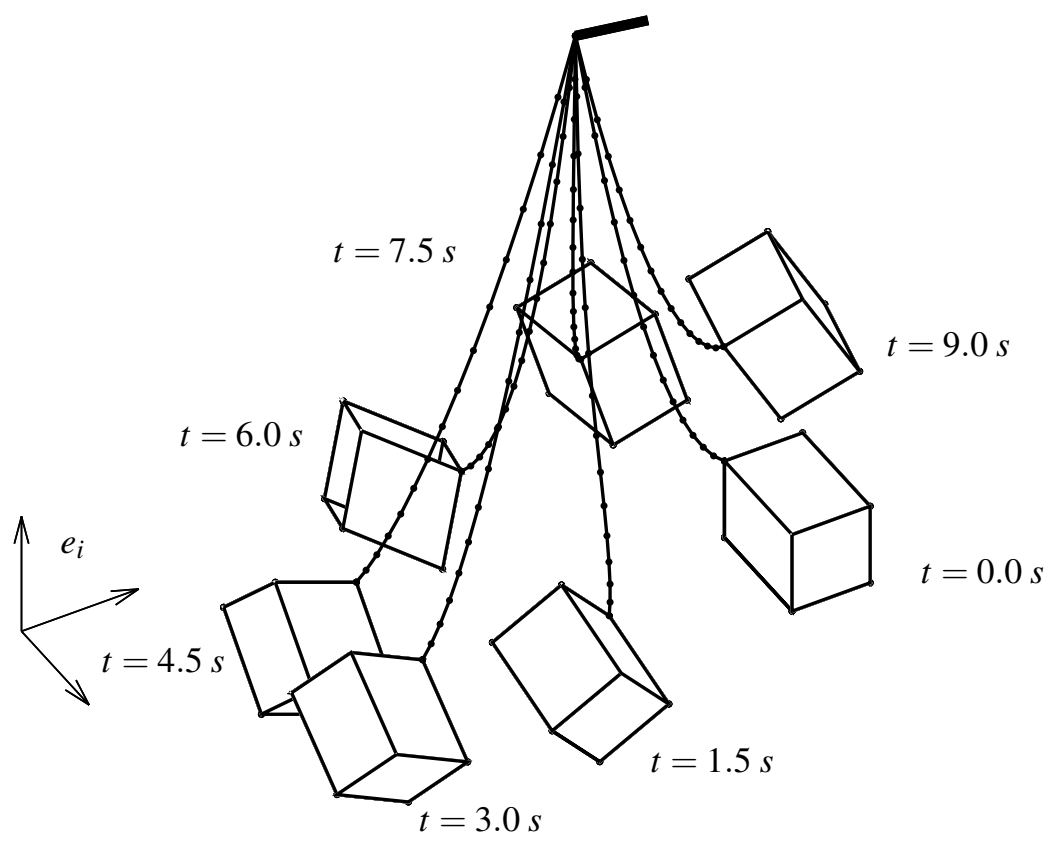

Figure 5. Free oscillation of a rigid cube with edge lenth $a=2$ and total mass $\bar{M}=0.1$ connected to a flexible rope with $\rho=1$ and $E=2$

\section{NUMERICAL EXAMPLE}

To verify the presented approach to the inverse dynamics problem under consideration, the following scenario is given. A rigid cube with edge length $a=2$ and mass $\bar{M}=1$ is supposed to accomplish a rest-to-rest maneuver. For this a translation of the cube from point $P_{0}=(0,0,0)$ to point $P_{f}=(2,2,2)$ along a straight line together with a simultaneous rotation of $\pi$ around the $\mathrm{Z}$-axis is planned. The maneuver is intended to start at $t_{0}=1.0 \mathrm{~s}$ and end at $t_{f}=9.0 \mathrm{~s}$. The motion of the rigid body described in terms of a Cosserat point subjected to geometric constraints can then be prescribed together with

$$
\varphi=-\frac{\pi}{2}\left(\cos \left(\frac{\pi}{2}(\sin \theta+1)\right)-1\right) \quad, \quad \theta=\pi\left(\frac{t-t_{0}}{t_{0}-t_{f}}-\frac{1}{2}\right)
$$


through the following trajectory:

$$
\gamma_{r b}=\left[\begin{array}{c}
0 \\
0 \\
0 \\
1 \\
0 \\
0 \\
0 \\
1 \\
0 \\
0 \\
0 \\
1
\end{array}\right] \quad \forall t<t_{0}, \quad \gamma_{r b}=\left[\begin{array}{c}
-\cos \varphi+1 \\
-\cos \varphi+1 \\
-\cos \varphi+1 \\
\cos \varphi \\
\sin \varphi \\
0 \\
-\sin \varphi \\
\cos \varphi \\
0 \\
0 \\
0 \\
1
\end{array}\right] \quad \forall t \in T_{m}=\left[t_{0}, t_{f}\right], \quad \gamma_{r b}=\left[\begin{array}{c}
2 \\
2 \\
2 \\
-1 \\
0 \\
0 \\
0 \\
-1 \\
0 \\
0 \\
0 \\
1
\end{array}\right] \forall t>t_{f}
$$

Thereby, four elastic ropes with mass density $\rho=1$ and Young's modulus $E=1$, are used to actuate the rigid body for which the motion is prescribed.
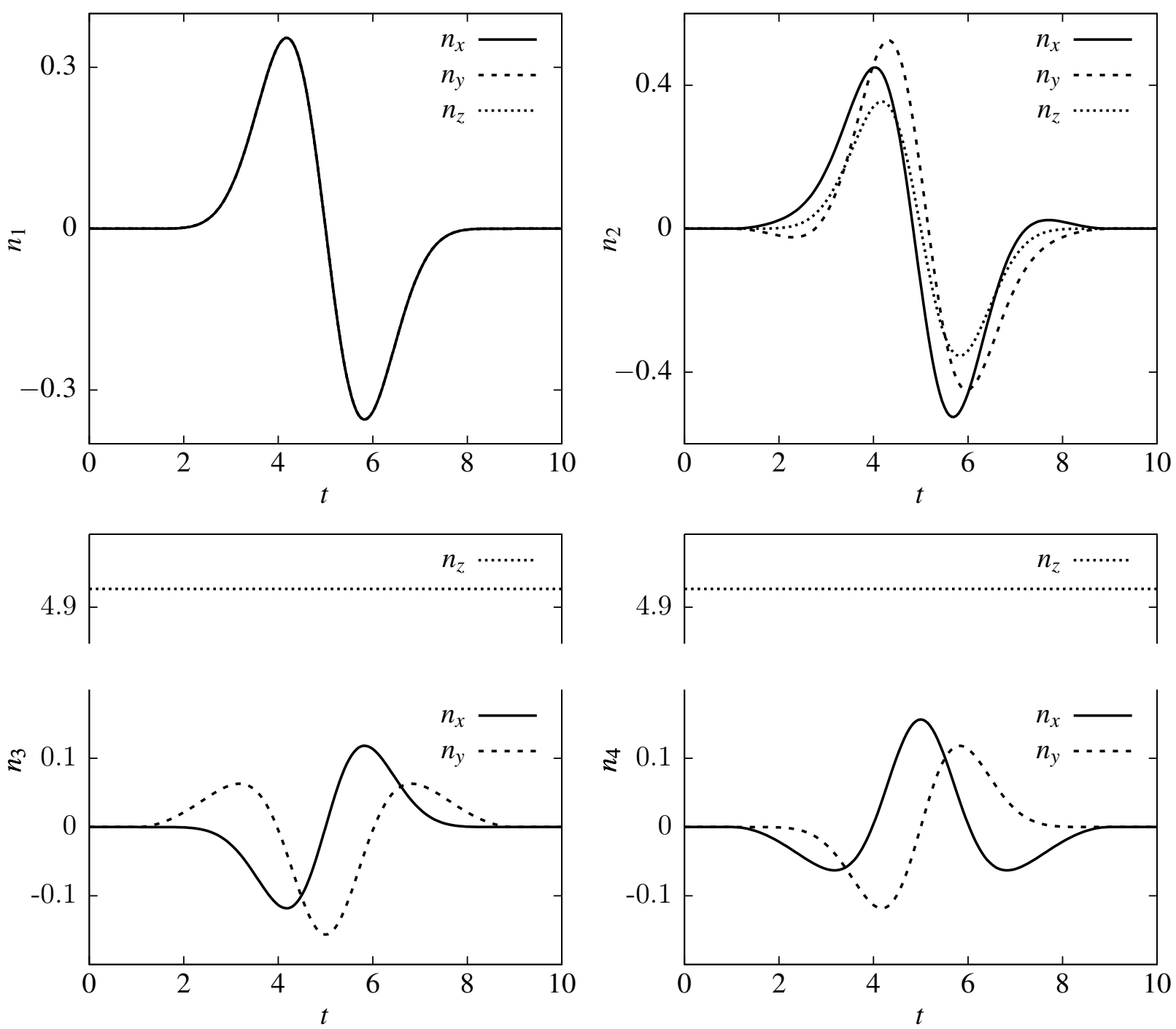

Figure 6. Components of the contact forces $n_{k}(t)$ such that the rigid body follows the prescribed translation of the rigid body from point $P_{0}=(0,0,0)$ to point $P_{f}=(2,2,2)$ accompanied by a simultaneous rotation $\pi$ 
Regarding the $k$ predefined contact points for $i \in\{1,2,3\}$

$$
P_{k}=\bar{x}+X_{i}^{k} d_{i}
$$

and following Section 3 the contact forces needed to achieve the desired motion can then be computed using (30) with

$$
H=\left[\begin{array}{cccc}
I & I & I & I \\
I & -I & I & -I \\
I & -I & -I & I \\
-I & I & I & I
\end{array}\right]
$$

where $I \in \mathbb{R}^{\alpha, \alpha}$ is the identity matrix. Note that, following Remark 3.1, the Lagrange multipliers cannot be left undefined. For the given maneuver, a uniaxial tension within the rigid body is choosen:

$$
\bar{\lambda}=\left[\begin{array}{llllll}
0 & 0 & \bar{M} g & 0 & 0 & 0
\end{array}\right]^{T}
$$

In Figure 6 components of the $k$ contact forces are shown. Subsequently, using the methods presented in Section 2 and Section 3, the forces acting on the upper end of the $k$ ropes at $s_{k}=0$ such that the rigid body at hand follows the prescribed trajectory, can be calculated. The numerical solution is shown in Figure 7.
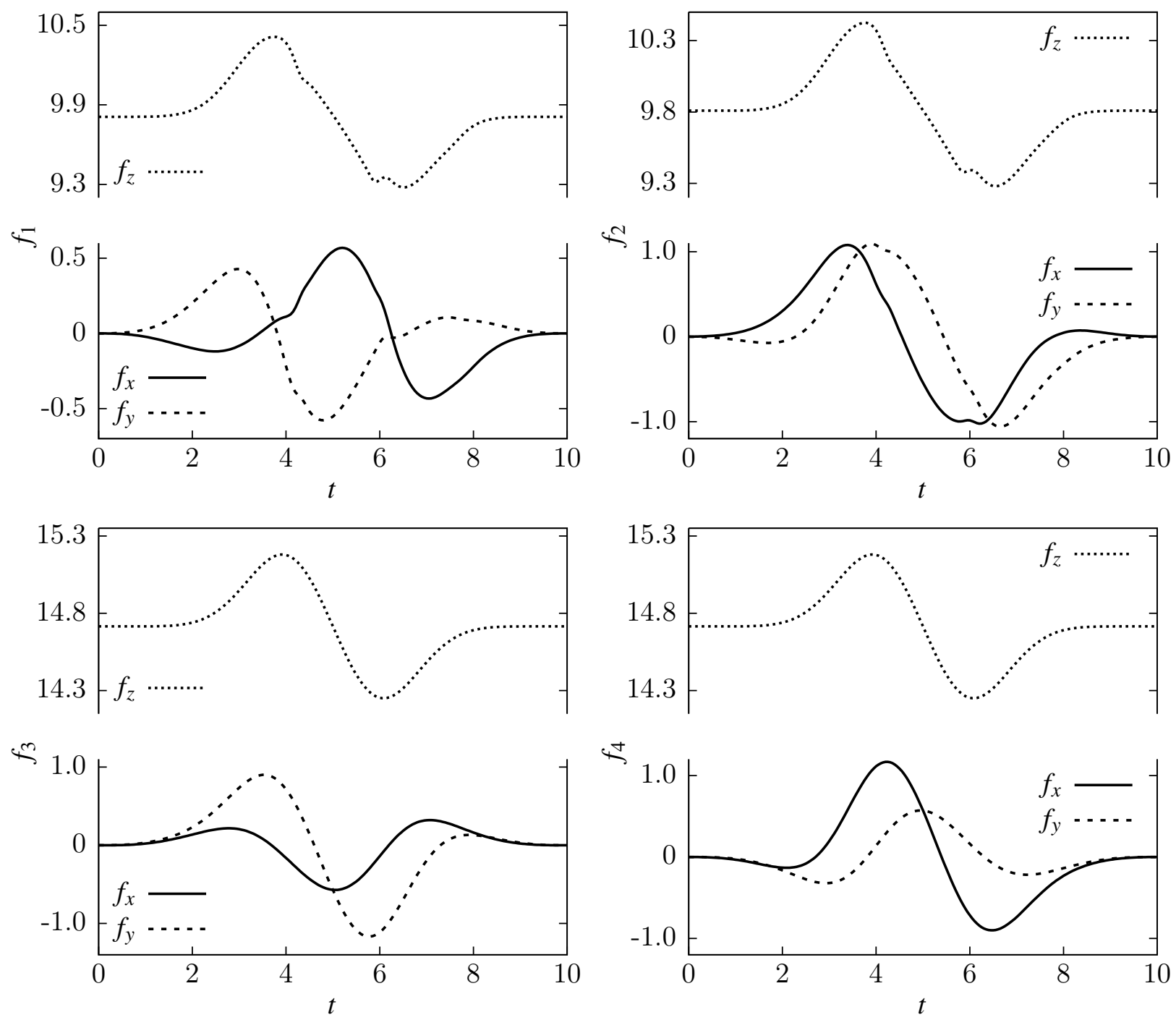

Figure 7. Components of the actuating forces $f_{k}$ acting on the upper end of the ropes at $s_{k}=0$ 
To verify the outlined method, the forces $f_{k}(t)$ can be inserted into the flexible multibody system presented in Section 4. In Figure 8 snapshots of a forward simulation of the flexible multibody system at hand actuated with the forces $f_{k}(t)$ acting at the upper ends of the $k$ ropes computed numerically using the approach presented above are shown.

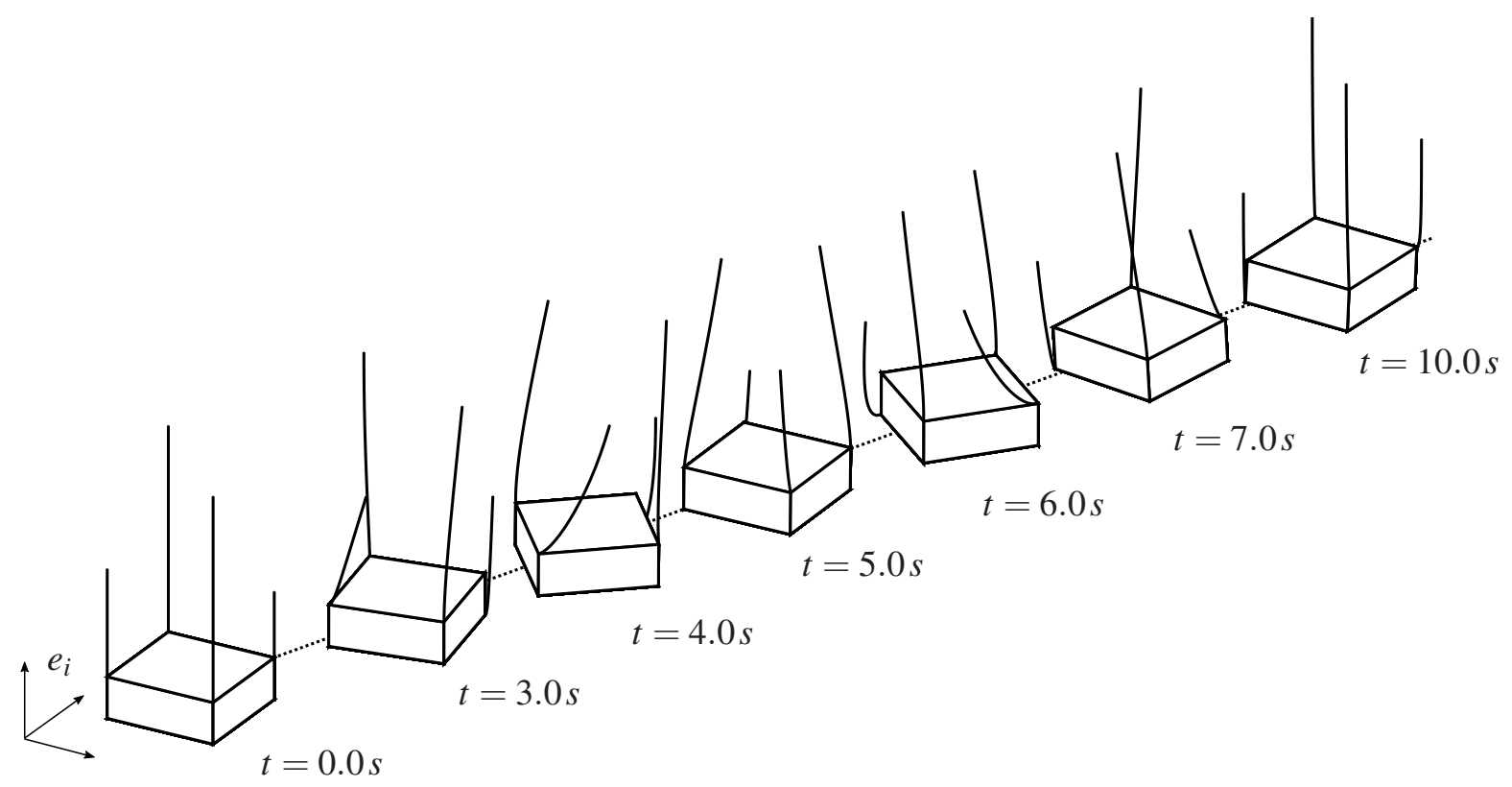

Figure 8. Snapshots of a rigid cube with edge length $a=2$ and total mass $\bar{M}=1$ actuated through four flexible ropes with $\rho=1$ and $E=2$ following a straight line from $P_{0}=(0,0,0)$ to $P_{f}=(2,2,2)$ while rotating simultaneously by the prescribed angle $\pi$

\section{CONCLUSIONS}

In this contribution the focus is laid on the cooperative control of a rigid body through flexible ropes. For this, the rigid body is supposed to follow any prescribed motion and an actuation is only achieved through flexible ropes attached to the rigid body at hand. Due to the differential flatness of the rope and the rigid body, the determination of the searched actuating forces, which are only allowed to act upon the upper ends of the attached ropes, can be carried out in a cascadelike manner. For this purpose, the rigid body is modelled as a Cosserat point under geometric constraints. By knowing the given motion of the body, the contact forces acting between the ropes and the rigid body can then be calculated directly from the resulting differential algebraic equation describing the motion of the body without integration. Now the task is to find the actuating force at the upper end of each rope, such that the geometric and static boundary conditions together with the partial differential equation governing the motion of the rope are fulfilled. The geometric and static boundary conditions are given by the prescribed motion of the lower end of the rope and the contact force, respectively. Common approaches, like the semi-discrete approach in which the underlying initial boundary value problem is discretised in space by applying appropriate methods and subsequently integrated in time, are not feasible for the control problem of flexible structures considered here. Therefore, a space-time finite element method is proposed in this contribution. This novel approach solves the control problem simultaneously in space and time and yields the actuating forces at the upper end of the ropes. Due to the cascade-like approach, the inverse computations of the rope-dynamics can be solved in parallel. The results of the inverse calculation of the actuating forces can then be verified by a forward simulation of a model of the considered flexible multibody system excited through the computed actuating forces. For this a direct model of 
the considered flexible multibody system is established in this contribution. This paper concludes by presenting a represenative numerical example.

\section{ACKNOWLEDGMENTS}

This work was supported by the Deutsche Forschungsgemeinschaft (DFG) under Grant BE 2285/121. This support is gratefully acknowledged.

\section{REFERENCES}

[1] Ströhle, T., Betsch, P.: Solution techniques for problems of inverse dynamics of flexible underactuated systems. In Kecskeméthy, A., Geu Flores, F., eds.: Multibody Dynamics 2019, Cham, Springer International Publishing (2020) 131-138

[2] Ströhle, T., Betsch, P.: Controlling nonlinear elastic systems in structural dynamics. In: WCCM-ECCOMAS2020, Paris, Virtual Congress, 14th World Congress on Computational Mechanics (WCCM) and ECCOMAS Congress (11-15 January 2021)

[3] Antman, S.S.: Nonlinear Problems of Elasticity. Volume 2. Springer (2005)

[4] Murray, R.M.: Trajectory generation for a towed cable system using differential flatness. IFAC Proceedings Volumes 29(1) (1996) 2792-2797 13th World Congress of IFAC, 1996, San Francisco USA, 30 June - 5 July.

[5] Altmann, R., Heiland, J.: Simulation of multibody systems with servo constraints through optimal control. Multibody System Dynamics 40 (2017) 75-98

[6] Argyris, J.H., Scharpf, D.W.: Finite elements in time and space. Nuclear Engineering and Design 10 (1969) 456-464

[7] Hughes, T.J.R., Hulbert, G.M.: Space-time finite element methods for elastodynamics: Formulations and error estimates. Computer Methods in Applied Mechanics and Engineering 66 (1988) 339-363

[8] Hesch, C., Schuß, S., Dittmann, M., Eugster, S.R., Favino, M., Krause, R.: Variational spacetime elements for large systems. Computer Methods in Applied Mechanics and Engineering 326 (2017) 541-572

[9] Betsch, P.: Energy-momentum integrators for elastic Cosserat points, rigid bodies, and multibody systems. In Betsch, P., ed.: Structure-preserving Integrators in Nonlinear Structural Dynamics and Flexible Multibody Dynamics, Cham, Springer International Publishing (2016) $31-89$

[10] Irscheid, A., Konz, M., Rudolph, J.: A flatness-based approach to the control of distributed parameter systems applied to load transportation with heavy ropes. In Kondratenko, Y.P., Chikrii, A.A., Gubarev, V.F., Kacprzyk, J., eds.: Advanced Control Techniques in Complex Engineering Systems: Theory and Applications: Dedicated to Professor Vsevolod M. Kuntsevich, Cham, Springer International Publishing (2019) 279-294 